\title{
PERCEPÇÕES DO COTIDIANO ALIMENTAR DE CRIANÇAS E ADOLESCENTES COM DIABETES MELLITUSTIPO 1
}

\author{
Renata Labronici Bertin ${ }^{1}$ \\ Nilce Priscila dos Santos Elizio ${ }^{2}$ \\ Raquel Nascimento Telles de Moraes ${ }^{3}$ \\ Caroline Opolski Medeiros ${ }^{4}$ \\ Lize Stangarlin Fiori ${ }^{5}$ \\ Anderson Zampier Ulbrich ${ }^{6}$
}

\begin{abstract}
RESUMO
0 diabetes mellitus tipo 1 (DM1) é responsável por 90\% dos casos de diabetes na infância, no entanto apenas 50\% dos casos são diagnosticados antes dos 15 anos. Atualmente a terapia nutricional tem sido fundamental na prevenção, tratamento e gerenciamento da doença, com vistas a proporcionar o bom estado nutricional, a saúde fisiológica e qualidade de vida do indivíduo. 0 objetivo deste estudo foi conhecer as percepcões e práticas alimentares de crianças e adolescentes portadores de DM1. A pesquisa teve caráter exploratório, com abordagem qualitativa, e voltou-se para crianças com idade entre 7 e 11 anos e com adolescentes com idade entre 12 e 18 anos. Foi realizada uma entrevista não estruturada com as mães das crianças menores de 11 anos, e com os próprios adolescentes, a partir de algumas questões norteadoras. As entrevistas foram examinadas pela técnica de análise de conteúdo. Os resultados evidenciaram que a doença não se instala apenas no corpo, mas ocupa um grande espaço na vida desses indivíduos e de seus familiares. Além disso, nota-se que as mães das crianças e os adolescentes estão atentas aos cuidados diários e enfrentam dificuldades no tratamento e controle do DM1, principalmente no que se refere à alimentação, aliadas aos aspectos emocionais, conviviais e socioeconômicos. Conclui-se que os profissionais da saúde devem buscar constantemente o conhecimento para que possam assistir o paciente diabético. Além disso, os nutricionistas devem criar estratégias que estimulem essa população na busca de uma alimentação adequada que proporcione a manutenção e promoção da saúde.
\end{abstract}

Palavras-chave: Diabetes Mellitus tipo 1. Criança. Adolescente. Hábitos alimentares.

PERCEPTIONS OF THE DAILY FOOD FOR CHILDREN AND ADOLESCENTS WITH TYPE 1 DIABETES MELLITUS ABSTRACT

Type 1 Diabetes Mellitus (DM1) is responsible for $90 \%$ of diabetes' cases in children, but only $50 \%$ of the cases are diagnosed before the age of 15 years old. The nutritional therapy is fundamental in prevention and treatment of the disease, in order to provide a good nutritional status, physiological health and individual life quality. The aim of the study was to understand the perceptions and eating habits of children and adolescents with type 1 . The research was exploratory, with a qualitative approach, with children between seven to eleven years old, and adolescents with ages between 12 to 18 years old. The semi-structured interview was done with the mothers of children under 11 years and with their own teenagers aged between 12 and 18 years old. Responses from the interviews were analyzed under the method of content analysis. It is evident that the disease does not only installs in the body, but occupies a large space in the lives of individuals and their families, in addition, it is noted that mothers of children and adolescents are aware of the daily care and face difficulties in treatment and control of DM1, especially when food is combined with the emotional, friendly and socioeconomic. It was concluded that health professionals must constantly search for knowledge that can help diabetes patients and professional nutritionist, strategies must be created in order to encourage the search for an adequate diet that provides the maintenance and promotion of health.

Keywords: Type 1 Diabetes Mellitus. Children. Adolescent. Food Habits.

\footnotetext{
${ }^{1}$ Nutricionista graduada pela Pontifícia Universidade Católica do Paraná - PUC/PR., doutora em Ciência dos Alimentos-UFSC, professora-adjunta do Departamento de Nutrição da Universidade Federal do Paraná-UFPR. rlbertin@yahoo.com 2 Nutricionista graduada pela Universidade Regional de Blumenau-Furb. priscilaelizio@yahoo.com.br Nutricionista graduada pela Universidade Regional de Blumenau-Furb. raquelntm@hotmail.com

${ }^{4}$ Nutricionista graduada pela Universidade Estadual do centro Oeste - Unicentro, doutora em Alimentos e Nutrição - FEA/Unicamp, professora-adjunta do Departamento de Nutrição da Universidade Federal do Paraná-UFPR. caroline.opolski@gmail.com

${ }_{5}^{5}$ Nutricionista graduada pelo Centro Universitário Franciscano, Unifra; doutora em Ciência e Tecnologia de Alimentos - UFSM, professora-adjunta do Departamento de Nutrição da Universidade Federal do Paraná-UFPR e professora da Universidade Positivo - UP. lizestangarlin@hotmail.com

${ }^{6}$ Educador Físico graduado pela Universidade Federal do Paraná-UFPR, doutor em Ciência do Movimento Humano, professor-adjunto do Departamento de Medicina da Universidade Federal do Paraná-UFPR. anderson_u@hotmail.com
} 
Diabetes mellitus (DM), segundo a terminologia da Sociedade Brasileira de Diabetes - SBD - não se trata de uma doença, mas sim de um grupo heterogêneo de distúrbios metabólicos que apresentam em comum a hiperglicemia, a qual pode ocorrer por defeito na ação da insulina e/ou na sua secreção (Sociedade..., 2015). Atualmente a classificação do DM baseia-se na etiologia e não no tipo de tratamento, e segundo a classificação proposta pela World Health Organization (WHO) (Alberti; Zimmer, 1998) e pela American Diabetes Association (ADA) (2014) tem-se quatro classes clínicas: DM tipo 1 (DM1), DM tipo 2 (DM2), outros tipos específicos de DM e DM gestacional (Sociedade Brasileira de Diabetes, 2015).

O DM1, foco do estudo, está presente em $5 \%$ a $10 \%$ dos casos e é caracterizado pela destruição de células betapancreáticas com consequente deficiência de insulina. Na maioria dos casos, essa destruição de células beta é mediada por autoimunidade, porém existem casos em que não há evidências de processo autoimune, sendo, portanto, referidos como forma idiopática de DM1 (Sociedade Brasileira de Diabetes, 2015).

Atualmente o DM1 é responsável por $90 \%$ dos casos de diabetes na infância, no entanto apenas $50 \%$ dos casos são diagnosticados antes dos 15 anos. Dados epidemiológicos, publicados no "Atlas de Diabetes 2013" da Federação Internacional de Diabetes (International Diabetes Federation - IDF) estimam uma prevalência de cerca de 500 mil crianças menores de 15 anos com DM1 no mundo. Entre os países com maior número de casos novos por ano estão Estados Unidos (13 mil), Índia (10.900) e Brasil (5 mil) (Patterson et al., 2014).

A infância e a adolescência são fases em que ocorre intenso desenvolvimento físico e psicológico dos indivíduos, assim como transformações biopsicossociais. A criança e/ou adolescente, além de lidarem com os aspectos próprios desses momentos, ao se defrontarem com uma doença crônica, como DM1, precisam enfrentar as demandas oriundas da doença e do tratamento, e com isso podem desenvolver desordens emocionais, como culpa, raiva, medo, angústia, depressão e apatia. Estas desor- dens, por sua vez, podem refletir no comportamento das crianças e adolescentes no ambiente escolar e familiar, bem como resultarem em uma maior dificuldade de adesão ao tratamento (Novato; Grossi; Kimura, 2008; Pilger; Abreu, 2007).

Um dos objetivos do tratamento do DM1 é a possibilidade de prevenção das complicações agudas e crônicas, haja vista o seu comprometimento na qualidade de vida de seus portadores. Nesse contexto, a ciência tem evidenciado que a terapia nutricional é fundamental na prevenção, tratamento e gerenciamento do DM1, tendo como objetivo proporcionar o bom estado nutricional, a saúde fisiológica e qualidade de vida do indivíduo, bem como prevenir e tratar complicações no curto e longo prazo e comorbidades associadas (American Diabetes Association, 2014; Lotemberg, 2008). Além disso, um programa de atividade física regular e um programa educacional completam o elenco do tratamento. Como se observa, para serem obtidos resultados satisfatórios com o tratamento é imprescindível uma cooperação muito estreita do trio "equipe médica, familiares, paciente" (Della Manna et al., 2002).

Diante do exposto, o presente estudo tem por objetivo conhecer as percepções e práticas alimentares de crianças e adolescentes portadores de DM1. A proposição deste texto justifica-se pela relevância dessa problemática no contexto global da saúde pública e por promover uma melhor compreensão acerca das dificuldades vivenciadas por crianças e adolescentes portadores de DM1. Ademais, discutir o significado do DM1 para a criança/adolescente e seus familiares proporcionará aos profissionais de saúde uma prática de cuidado mais qualificada e com maior resolutividade.

\section{PROCESSO TEÓRICO-METODOLÓGICO}

A fim de proceder a uma análise crítico-interpretativa acerca dos saberes e práticas do cotidiano alimentar de crianças e adolescentes portadores de DM1, desenvolveu-se uma pesquisa de cunho qualitativo de caráter exploratório. Segundo Triviños 
(2009), essa abordagem responde a questionamentos extremamente particulares, além de oferecer a possibilidade de conhecer a realidade pela percepção, vivência, experiência de vida e reflexão a fim de transformá-la. Para o autor, a pesquisa qualitativa parte da descrição dos fenômenos, procurando captar não só a sua aparência como também a sua essência. Este texto busca as causas, a origem, as relações, as mudanças e suas consequências para a vida humana. Considerando esta perspectiva, o estudo voltou-se para crianças e adolescentes portadores de DM1, de ambos os sexos, atendidos em um Ambulatório Universitário da cidade de Blumenau-SC.

Os participantes desta pesquisa foram identificados por meio dos prontuários encaminhados pelo médico endocrinologista responsável pelo atendimento, e selecionados a partir dos seguintes critérios de inclusão: ter sido diagnosticado com DM1 há pelo menos um (1) ano, ser cadastrado no Ambulatório Universitário, com idade entre 7 e 18 anos e ter participação mensal no grupo de diabetes mellitus - no ano de 2009. As crianças e adolescentes foram convidados a participar da pesquisa durante a reunião mensal do grupo de diabetes, que se realizava uma vez por mês no Ambulatório Universitário.

O número de participantes foi considerado suficiente quando os dados da pesquisa refletiram a totalidade das múltiplas dimensões do objeto deste estudo e se tornaram repetitivos (Minayo, 2007). Assim, participaram do estudo dez portadores de DM1, entre crianças e adolescentes.

A coleta de dados foi realizada por um dos autores, em uma sala reservada para esta finalidade dentro do Ambulatório Universitário, após a leitura dos objetivos da pesquisa e assinatura do Termo de Consentimento Livre e Esclarecido (TCLE), por meio de uma entrevista não estruturada com as mães das crianças com idade entre 7 e 11 anos e com os próprios adolescentes entre 12 e 18 anos. As questões que nortearam a entrevistas são descritas a seguir: "Após o diagnóstico de DM1, qual a principal dificuldade apresentada pelo seu filho(a) e/ou por você em relação a essa doença?”; "De uma forma geral, fale sobre o cotidiano alimentar de seu filho(a) e/ou seu com DM1"; "Foram inseridos na alimentação do seu filho(a) e/ou na sua, alimentos recomendados para portadores de DM1?"; "Como é a alimentação do seu filho(a) e/ou a sua, em um ambiente não domiciliar?"; "Como foi o envolvimento da família no cotidiano do seu filho e/ou no seu cotidiano?"

As entrevistas foram gravadas e, em seguida, transcritas na íntegra, o que possibilitou o registro fidedigno de todas as informações fornecidas pelos entrevistados. Os participantes foram identificados mediante algarismos romanos de I a X, conforme a ordem das entrevistas.

Para a análise dos dados, foi utilizada a técnica de análise de conteúdo, seguindo metodologia proposta por Bardin (2004).

Ressalta-se que todas as etapas deste estudo respeitaram os preceitos éticos da pesquisa envolvendo seres humanos, tendo sido o projeto aprovado pelo Comitê de Ética em Pesquisa da Fundação Universidade Regional de Blumenau, sob n 072/09.

\section{RESULTADOS E DISCUSSÃO}

As crianças e os adolescentes entrevistados totalizaram 4 meninas e 6 meninos com idade média de 12,75 anos $\pm 3,6$ e 15,75 anos $\pm 1,5$ respectivamente. A análise das entrevistas revelou as experiências das crianças e adolescentes com o seu cotidiano, bem como os valores emocionais, psicológicos, econômicos e sociais que carregam consigo e que indiretamente afetam o dia a dia de toda uma estrutura familiar. A análise de conteúdo permitiu a construção de categorias analíticas, a saber: diagnóstico do DM1 e suas repercussões; inserção dos alimentos recomendados para diabéticos; alimentação em ambiente não domiciliar e vivência da família no cotidiano das crianças e adolescentes com DM1, as quais serão apresentadas a seguir. 


\section{DIAGNÓSTICO DE DMI E SUAS REPERCUSSÕES}

A partir da pergunta "Quais as principais dificuldades encontradas após o diagnóstico da DM1”? torna-se evidente na fala dos sujeitos as dificuldades no controle da dieta e a reestruturação alimentar na nova rotina. Sabe-se que uma das estratégias de tratamento do DM1 é orientar paciente/familiar sobre o planejamento alimentar diante da aplicação das insulinas, bem como orientar sobre a necessidade de se evitar o consumo de alguns alimentos e/ou adaptá-los aos horários das refeições (Sociedade..., 2015).

Não poder comer doce (I, II, VI, IX).

[...] é mais a contagem de carboidrato, e a dificuldade de saber a quantidade de cada coisa que eu vou comer $(\mathrm{V})$.

[...] se a gente vai ver, todo o produto tem alguma coisa com açúcar, se não é açúcar em si, é alguma coisa que vai açúcar, ou alguma coisa que se transforma em açúcar no corpo, porque mesmo o arroz, feijão e macarrão, isso eu não posso comer demais, porque é carboidrato e carboidrato vai alterar minha glicose $(\mathrm{X})$.

Em estudo realizado por Zanetti e Mendes (2001), pode-se observar que a alteração na dieta foi a principal dificuldade relatada pelas mães de crianças e adolescentes com DM1.

A pesquisa conduzida por Moreira e Dupas (2006) também revela o esforço das crianças em habituar-se a uma nova vida, argumentando o controle da doença nos seus diversos âmbitos, principalmente no que se refere à dieta.

Em estudo realizado por Leal et al. (2012), que avaliaram a vivência dos familiares de crianças e adolescentes portadores de DM1, foi constatado que, por grande parte dos familiares, a reestruturação alimentar voltada para o controle dos hábitos alimentares e restrição de alguns alimentos, principalmente doces, ainda é uma barreira a ser vencida.
Outro fator que foi contemplado nas falas dos sujeitos em relação às dificuldades encontradas refere-se principalmente às mudanças cotidianas da alimentação, como demonstrado a seguir:

A gente tentou cortar o máximo possível de entrada de bolo, essas coisas doces, tentamos nesse lugar colocar integrais, alguma coisa, não muito, por causa do acesso, que é um pouco caro. Então a gente tá fazendo o máximo possível (IV).

Eu não gostava muito de comer verdura e essas coisas assim, daí quando eu descobri a diabetes [...], agora eu como todo dia $(\mathrm{V})$.

Ah, com certeza, na alimentação como eu comia bastante doce [...] tudo teve que acabar assim, de uma hora para outra $(\mathrm{X})$.

A alimentação é parte fundamental do tratamento de todas as pessoas com diabetes e deve ser entendida como um planejamento cuidadoso e balanceado, mas nunca como uma simples listagem de proibições e limitações alimentares. Deve ser individualizada de acordo com as condições sociais, econômicas e culturais, bem como estar em harmonia com a rotina diária da pessoa, apesar de existir uma série de orientações gerais a serem consideradas (Ribeiro et al., 2006). Assim, a criança e o adolescente tentam se adaptar às necessidades impostas pela doença, aprendendo a conviver com as restrições alimentares, controlando a quantidade de determinados alimentos, escolhendo outros mais saudáveis para inserir na alimentação cotidiana (Moreira; Dupas, 2006).

Também foram contempladas nas falas dos sujeitos questões relacionadas ao próprio tratamento da doença, a qual requer cuidado e atenção, principalmente em relação à terapia insulínica (aplicação e ajustes das doses) e a monitoração da glicemia. Segundo Maia e Araújo (2004), os indivíduos portadores de uma patologia crônica sentem-se cansados pelo inconveniente da realização diária do tratamento.

É tomar o remédio e tal [...] (VI).

É fazer a insulina, e fazer o exame da glicemia, que é todo dia, de manhã e à noite (VIII). 
[...] foi bem difícil, porque eu nunca apliquei nada em mim mesmo, seringa pra mim era só duas vezes ao ano, [...], daí no começo era minha mãe que me aplicava, mas era complicado, não podia depender dela pra sempre, depois [...] comecei a aplicar em mim mesmo [...] (X).

Para muitos indivíduos, a constante necessidade de automonitorização e as aplicações diárias de insulina podem ser extremamente desconfortáveis, frustrantes e preocupantes, levando muitas vezes a omissões de doses de insulina, com maior incidência de complicações agudas graves.

Em pesquisa realizada por Grossi, Cianciarullo e Manna (2002), os autores verificaram que o desconforto psicossocial gerado pela doença possui impacto negativo sobre a capacidade do paciente de iniciar e manter as recomendações básicas de automonitorização. Com isso, há um comprometimento da qualidade de vida do paciente, o qual apresenta menor adesão ao tratamento, piora do controle glicêmico e maior número de complicações em longo prazo.

\section{INSERÇÃO DOS ALMENTOS RECOMENDADOS PARA DIABÉTICOS}

Nesta categoria perguntou-se aos participantes as principais dificuldades encontradas para inserir alimentos recomendados para portadores de diabetes, e a maioria relatou que os produtos recomendados para diabéticos, dentre eles os diet e integrais, são vendidos a um valor alto, comparado aos produtos normais. Com isso o acesso a estes alimentos torna-se difícil e limitado por boa parte dos portadores de DM1, pois nem sempre as famílias têm condições financeiras para sua aquisição.

A situação financeira entre os participantes do nosso estudo não é diferente desta realidade. Praticamente todos os integrantes tinham condições similares e apresentavam dificuldades em relação aos recursos financeiros para o cuidado e a manutenção do tratamento.
[...] convenhamos, produtos diet ou light não são tão acessíveis assim e eu não tenho como ficar comprando todo mês ou toda vez que precisa (II).

[...] o maior problema hoje ainda é o financeiro, porque esses produtos são muito mais caros do que o normal (IV).

[...] o wafer diet é três reais, imagina, o tradicional é noventa centavos (IX).

[...] achar não é difícil, o problema tá na diferença de preço entre um produto diet e do que não é $\operatorname{diet}(\mathrm{X})$.

Para De Mello (2006), a condição financeira tem repercussões na qualidade do tratamento. Em uma pesquisa realizada por Peres, Franco e Santos (2006), os aspectos financeiros foram considerados um dos itens que dificultavam o seguimento da dieta por portadores de diabetes. A boa condição financeira foi vista como um facilitador e um estímulo para seguir a alimentação recomendada. Resultados semelhantes foram encontrados por Fragoso et al. (2010) ao observarem que a baixa renda familiar dificultava a oferta de uma dieta correta a adolescentes portadores de DM1.

Em estudo realizado por Souza (2005) é possível observar que a renda é um fator determinante no consumo desses produtos, os quais esses são geralmente mais caros do que os tradicionais, e muitos consumidores não têm renda suficiente para adquiri-los esses produtos com frequência. Para alguns participantes, os produtos diet e light tem um alto custo, sendo necessário comparar preços e escolher o mais barato.

Outro estudo realizado por Hall (2006) também mostrou que a renda influencia no consumo de produtos diet e light, pois quanto mais alta é a renda, maior é o consumo desses produtos.

Algumas falas mostram que uma das dificuldades para inserir os alimentos recomendados para diabéticos é o sabor destes produtos, porém em outras falas este aspecto não foi considerado um problema.

Ah, ele tem um gosto diferente, tanto que eu prefiro o gosto do normal (III). 
Não pelo sabor, porque são ótimos, ele se adaptou legal [...] (IV).

O gosto, a maioria tem um gosto. Não que seja ruim, entende. Mas é que, os que não são diet têm um gosto melhor, mas não que o diet seja ruim $(\mathrm{X})$.

A qualidade sensorial do alimento (sabor, cheiro, textura, aparência) relaciona-se às características e às condições fisiológicas, psicológicas e sociológicas do indivíduo que ingere os alimentos, sendo fator determinante do comportamento e escolha alimentar (Assis; Nahas, 1999).

$\mathrm{Na}$ pesquisa realizada por Souza (2005) a diferenciação no sabor dos produtos dietéticos em comparação com os naturais foi destacada como um fator que desestimula os consumidores na compra dos produtos. Os entrevistados deste estudo relataram não consumir produtos diet e light, por não gostarem do sabor ou considerarem o preço elevado.

Segundo Peres et al. (2006), algumas mulheres portadoras de DM2 relataram não usar adoçante, pelo fato de não se acostumarem com o sabor, pois não é tão saboroso como o açúcar normal.

Já entre portadores de DM pesquisados por Casto e Franco (2002), os autores verificaram que os portadores de DM gostavam dos adoçantes porque se acostumaram com o seu uso, demonstrando assim que a ingestão de um novo alimento no plano alimentar pode mudar o hábito e fazer com que o paciente se adapte à nova situação de consumo.

\section{ALIMENTAÇÃO EM AMBIENTE NÃO DOMICILIAR: Dificuldades em Relação às Refeições Servidas}

Foi indagado aos participantes como era a alimentação em ambiente não domiciliar, com o intuito de conhecer e compreender as dificuldades alimentares das crianças e/ou adolescentes quando estão em festas, casas de amigos e até mesmo na escola.
Constatou-se durante a entrevista que alguns participantes levam seu próprio alimento quando não estão em um ambiente domiciliar.

Eu levo junto uma coisinha, uma bolacha, coisa diet eu posso comer $[\ldots](\mathrm{V})$.

[...] já levo o meu refrigerante, aí tem brigadeiro, daí fala não pode comer, um só pra matar aquele desejo, mas se não fica só no salgado [...] (VII).

[...] eu sempre levo alguma coisa para comer junto. Ah, em festa eu como só coisa salgada [...] (VI).

Realizar a dieta recomendada reveste-se de vários significados, como a perda do prazer de comer e beber, restrição da autonomia e cerceamento da liberdade para se alimentar como e quando desejar. As proibições impostas pela doença e seu tratamento limitam a liberdade de fazer o que se tem vontade (Peres; Franco; Santos, 2006).

A doença acarreta mudanças significativas na relação que o paciente diabético estabelece com seu próprio corpo e com o mundo que o cerca, e isso se traduz principalmente no aspecto alimentar. Na terapêutica do diabetes é fundamental cumprir a dieta. $\mathrm{O}$ ato de comer, porém, é complexo e não remete apenas à ingestão de nutrientes, mas também a uma gama de emoções e sentimentos, além de valores culturais, o que torna árduo o cumprimento das recomendações (Santos; Enumo, 2003).

Diferentemente da subcategoria anterior, alguns participantes se excluem do convívio social, de alguns eventos, por não se sentirem bem.

[...] ele prefere não ir, porque daí ele não pode comer o bolo, que é a coisa que ele mais gosta (VIII).

Ele nem vai, [...] porque daí lá ele não vai poder comer o que os amigos dele comem (VIII).

[...] na casa de amigos ele não vai (IX).

De acordo com Luescher e Beraldo (2004), a alimentação não deve aprisionar a criança e/ou adolescente, e a família deve estar preparada para os dias de festa, educada para realizar teste de glicemia capilar antes e depois da comemoração e para evitar ou corrigir possíveis hiperglicemias, com uso de 
insulina de ação rápida. De acordo com os autores, as crianças e os adolescentes não devem ser afastados da sociedade e devem participar de eventos sociais, como festas de aniversário de amigos e colegas, sem colocar em risco sua saúde.

Em relação à escola, ficou evidenciado nas falas a seguir o quanto as adaptações, necessárias em decorrência da patologia, repercutem no âmbito escolar. Observa-se e evidencia a necessidade de adaptação da alimentação e de atividades rotineiras impostas pela doença para a criança e o adolescente diabético.

Eles dão o lanche lá na escola, já foi falado, daí quando a merenda não é salgada, eles dão tudo pra ele, eles dão um copo de leite, bolacha salgada, então eles já sabem, tratam meu filho diferente (VIII).

O colégio sabe tudo sobre os cuidados que devem ter com a alimentação do meu filho (IV).

Quando a minha glicose está baixa eu começo a tremer, aí chamo a professora e ela deixa eu comer meu lanche dentro da sala mesmo (VII).

A convivência com o diabetes pode ser tranquila a ponto de não atrapalhar a vida dos adolescentes, apesar da presença de algumas dificuldades em relação à escola e ao trabalho e da preocupação constante dos pais em relação aos cuidados com a doença (Santos; Enumo, 2003).

Os pais preocupam-se em saber como o professor do filho reconhecerá os sinais e sintomas da hipoglicemia para ajudá-lo na hora do tratamento. Por outro lado, sabe-se que os professores podem não ter ou possuir pouco conhecimento e/ou experiência sobre DM1 (Zanetti; Mendes, 2001).

Cabe destacar que apesar das dificuldades alimentares sofridas pelas crianças e adolescentes diabéticos o tratamento deve ser multiprofissional, quando profissionais da saúde e da escola, junto com os familiares, trabalhem para que a criança e o adolescente saibam lidar com a rotina da dieta e insulina, melhorando a sua qualidade de vida (Zanetti; Mendes, 2001).

\section{VIVÊNCIA DA FAMÍLLA NO COTIDIANO DAS CRIANÇAS E ADOLESCENTES COM DMI}

Nesta categoria perguntou-se aos participantes sobre o conhecimento adquirido pela família após a descoberta da doença da criança ou do adolescente e o que isso agregou para um melhor convívio com o problema, tanto do diabético quanto dos familiares. De um modo geral, as falas destacam que a organização da família é fundamental para a execução efetiva das tarefas relacionadas ao controle do diabetes, além de ajudar essas crianças e esses adolescentes a aceitarem sua condição de saúde e a viverem com uma melhor qualidade de vida.

Minha mãe ia comigo participar das atividades do ambulatório de diabetes, assistir as palestras sobre alimentação saudável, exercício físico e tudo mais (I).

A minha mãe ela já prefere deixar tudo igual lá em casa, todos comem a mesma comida para que eu não me sinta diferente dos outros de minha casa (VII).

No primeiro ano que eu apresentei diabetes, toda minha família perguntou como eu me sentia, perguntaram como era minha alimentação, como era minha rotina $[. .$.$] (VIII).$

[...] eu vejo que minha mãe prepara a alimentação para que eu possa escolher alguma coisa, daí ela sempre prepara alguma coisa que eu possa comer com eles ou alguma coisa que não tenha tanto açúcar, pra não comer separado, porque é ruim a gente pensar que é diferente dos outros, porque tem de comer alguma coisa separada (IX).

[...] todo mundo ali em casa pelo menos, quando eu tava no hospital, já haviam perguntado ao médico sobre o que era a doença, e quais cuidados teriam que ter comigo $(\mathrm{X})$.

As informações referentes à DM1 promovem o aprimoramento de habilidades e favorecem a autoconfiança da família e das crianças e adolescentes com a doença. O conhecimento leva à compreensão sobre a condição crônica de saúde causada pela doença e como isso pode influenciar no futuro (De Mello, 2006). 
É importante ressaltar que a proteção familiar, além de resguardar, abrigar e preservar a vida e a saúde de seus membros, também significa sua orientação e acompanhamento, principalmente nessas fases tão importantes e marcantes da vida, passagem da infância/adolescência para a vida adulta. Nessas fases, geralmente eles não se conformam com as restrições que o tratamento da doença impõe. Algumas famílias verbalizaram claramente que a preocupação diante da situação de saúde dos seus filhos é de que eles um dia possam assumir seu autocuidado, tanto na aplicação de insulina quanto nos cuidados com a alimentação, atividade física e os testes de glicemia capilar (Mattosinho; Silva, 2007; De Mello, 2006).

Neste contexto, o papel da família torna-se importante para que a criança e/ou adolescente possam manter o equilíbrio emocional. A maneira como a família lida com a situação influenciará a criança e o adolescente na aceitação ou negação da doença. Para tanto, é necessário que os pais sejam compreensivos, sem superproteger. Na medida do possível, eles devem evitar acentuar as diferenças entre o diabético e os outros integrantes da família, para que a criança e o adolescente, diabéticos não se sintam discriminados (Fialho et al., 2011).

Por fim, cabe destacar que pessoas que dispõem de um bom arcabouço familiar acabam tendo muito mais condições de enfrentar o diabetes e sair-se bem no seu controle, além de conseguirem que a doença tenha o menor impacto possível em sua vida (Fialho et al., 2011).

\section{CONSIDERAÇÕES FINAIS}

Ao mostrarmos algumas percepções de mães de crianças e adolescentes, que vivenciam a condição crônica do DM1, pode-se compreender que a doença não se instala apenas no corpo, mas ocupa um grande espaço na vida desses indivíduos e de seus familiares, modificando-a em muitos aspectos, além de causar conflitos que provêm do tratamento da doença.
Foi possível perceber que as mães das crianças e os adolescentes estão atentos aos cuidados diários e enfrentam dificuldades no tratamento e controle do DM1, principalmente no que se refere à alimentação, aliada aos aspectos emocionais, de convivência social e condição socioeconômica.

Diante da realidade exposta, constata-se a importância da promoção de educação nutricional entre crianças e adolescentes, com ênfase para a escolha apropriada dos alimentos e seu papel para a saúde e melhoria do tratamento.

Com base nesses pressupostos entende-se que a integralidade da atenção voltada aos portadores de diabetes precisa ser posta em movimento pelos profissionais de saúde em suas práticas de cuidado, pois dela depende a possibilidade de um enfrentamento menos sofrido dessa experiência e do abrir-se para outras formas de viver a vida.

Desta maneira, ressalta-se que os profissionais da saúde devem buscar constantemente o conhecimento para que possam assistir o paciente diabético, e especialmente o profissional nutricionista deve criar estratégias que estimulem esta população na busca de uma alimentação adequada e saborosa, proporcionando a manutenção e promoção da saúde, assim como uma melhora na qualidade de vida desses indivíduos.

\section{REFERÊNCIAS}

ALBERTI, K. G.; ZIMMER, P. Z. Definition, diagnosis and classification of diabetes mellitus and its complications. 1. Diagnosis and classification of diabetes mellitus provisional report of a WHO consultation. Diabetic Med, v. 15, n. 7, p. 539-553, 1998.

AMERICAN DIABETES ASSOCIATION. Standards of Medical Care in Diabetes. Diabetes Care, v. 37, n. Suppl 1, p. 81-90, 2014.

ASSIS, M. A. A.; NAHAS, M. V. Aspectos motivacionais em programas de mudanças de comportamento alimentar. Rev. Nutr., v. 12, n. 1, p. 33-41, 1999. 
BARDIN, L. Análise de conteúdo. 3 ed. Lisboa: Edições 70, 2004.

CASTO, A. G. P.; FRANCO, L. J. Caracterização do consumo de adoçantes alternativos e produtos dietéticos por indivíduos diabéticos. Arq Bras Endocrinol Metab, v. 46, n. 3, p. 280-287, 2002.

DE MELLO, M. B. Déficits de autocuidado vivenciados por famílias de crianças e adolescentes com Diabetes mellitus tipo 1. 2006. 90 f. Dissertação (Mestrado em Enfermagem) - Universidade Federal de Santa Catarina, Florianópolis, 2006.

DELLA MANNA, T. et al. Diabetes mellitus na infância e adolescência. In: SETIAN, N. Endocrinologia pediátrica - aspectos físicos e metabólicos do recém-nascido ao adolescente. São Paulo: Sarvier, 2002.

FIALHO, F. A. et al. Crianças e adolescentes com diabetes mellitus: cuidados/complicações para a enfermagem. Revista Baiana de Enfermagem, v. 25, n. 2, p. 145-154, 2011.

FRAGOSO, L. V. C. et al. Vivências cotidianas de adolescentes com diabetes mellitus tipo 1. Texto Contexto Enferm, Florianópolis, v. 19, n. 3, p. 443451, 2010.

GROSSI, S. A. A.; CIANCIARULLO, T. I.; MANNA, T. D. Avaliação de dois esquemas de monitorização domiciliar em pacientes com diabetes mellitus do tipo 1. Rev. Esc. Enferm. USP, , v.36, n. 4, p. 317-323, 2002.

HALL, R. J. Fatores que influenciam o consumo de produtos diet e light no Brasil. 2006. 102f. Dissertação (Mestrado em Agronegócios) - Universidade Federal do Mato Grosso. Campo Grande, 2006.

LEAL, D. et al. A vivência dos familiares de crianças e adolescentes portadores de diabetes mellitus tipo 1. Revista Eletrônica de Enfermagem, v. 14, n. 1, p. 189-196, 2012.

LOTTEMBERG, A. M. P. Características da dieta nas diferentes fases da evolução do diabetes melito tipo 1. Arq Bras Endocrinol Metab, São Paulo, v. 52, n. 2, p. 250-259, 2008.

LUESCHER, J. A.; BERALDO, R. S. Diabetes em crianças. In: OLIVEIRA, J. E. P.; MILECH, A. Diabetes mellitus: clínica, diagnóstico, tratamento multidisciplinar. São Paulo: Atheneu, 2004.
MAIA, F. R.; ARAUJO, L. R. Aspectos psicológicos e controle glicêmico de um grupo de pacientes com Diabetes Mellitus tipo 1 em Minas Gerais. Arq Bras Endocrinol Metab, São Paulo, v. 48, n. 2, p. 261-266, 2004.

MATTOSINHO, M. M. S.; SILVA, D. M. G. V. Itinerário terapêutico do adolescente com Diabetes mellitus tipo 1 e seus familiares. Rev. Latino-Am. Enfermagem, v. 15, n. 6, p. 1113-1119, 2007.

MINAYO, M. C. S. O desafio do conhecimento: pesquisa qualitativa em saúde. 10. ed. São Paulo: Hucitec, 2007.

MOREIRA, P. L.; DUPAS, G. Vivendo com o diabetes: a experiência contada pela criança. Rev. Latino-am. Enfermagem, v. 14, n. 1, p. 25-32, 2006.

NOVATO, T. S.; GROSSI, S. A. A.; KIMURA, M. Qualidade de vida e auto-estima de adolescentes com diabetes mellitus. Acta Paulista de Enfermagem, v. 21, n. 4, p. 562-567, 2008.

PATTERSON, C. et al. Diabetes in the young - a global view and worldwide estimates of numbers of children with type 1 diabetes. Diabetes Res Clin Pract, v. 103, n. 2, p. 161-175, 2014.

PERES, D. S, P.; FRANCO, L. J.; SANTOS, M. A. Comportamento alimentar em mulheres portadoras de diabetes tipo 2. Rev. Saúde Pública, v. 40, n. 2, p. 310-317, 2006.

PILGER, C.; ABREU, I.S. Diabetes mellitus na infância: repercussões no cotidiano da criança e de sua família. Cogitare Enfermagem, v. 12, n. 4, p. 494-501, 2007.

RIBEIRO, M. R. et al. Diabetes mellitus. In: LOPES, C. Tratado de Clínica Médica, São Paulo: Medica Missau, 2006. V. 2.

SANTOS, J. R.; ENUMO, S. R. F. Adolescentes com diabetes mellitus tipo 1: seu cotidiano e enfrentamento da doença. Psicologia: Reflexão e Crítica, v. 16, n. 2, p. 411-425, 2003.

SOCIEDADE BRASILEIRA DE DIABETES. SBD. Diretrizes da Sociedade Brasileira de Diabetes: 2014-2015. São Paulo, AC Farmacêutica, 2015. Disponível em: <http://www.diabetes.org.br/ images/2015/area-restrita/diretrizes-sbd-2015.pdf $>$. Acesso em: 10 ago. 2015. 
SOUZA, E. P. Comportamento do consumidor de produtos diet e light na cidade de Fortaleza - Ceará. CONGRESSO BRASILEIRO DE ECONOMIA E SOCIOLOGIA RURAL, 2005. Ribeirão Preto. Anais... Brasília, 2005.

TRIVIÑOS, A. N. S. Introdução à pesquisa em ciências sociais: a pesquisa qualitativa em educação. São Paulo: Atlas, 2009.

YISAHAK, S. et al. Diabetes in North America and The Caribbean: An update. Diabetes Research and Clinical Practice, v. 103, n. 2, p. 223-230, 2014.

ZANETTI, M. L.; MENDES, I. A. C. Análise das dificuldades relacionadas às atividades diárias de crianças e adolescentes com diabetes mellitus tipo 1: depoimento das mães. Rev. Latino-Am. Enfermagem, Ribeirão Preto, v. 9, n. 6, p. 25-30, 2001.

Recebido em: 21/12/2015

Aceito em: 25/4/2016 